\title{
0 perfil da sexualidade em homens com lesão medular
}

\section{Profile of sexuality in men with spinal cord injury}

\author{
Larissa Amaral Torrecilha ${ }^{[a]}$, Bianca Teixeira Costa ${ }^{[b]}$, Fellipe Bandeira Lima ${ }^{[c]}$, \\ Suhaila Mahmoud Smaili Santos ${ }^{[\mathrm{d}]}$, Roger Burgo de Souza ${ }^{[\mathrm{e}]}$
}

[a] Residente em Fisioterapia Neurofuncional - Adulto no Hospital Universitário Regional do Norte do Paraná, Universidade Estadual de Londrina (UEL), Londrina, PR - Brasil, e-mail: lari_torrecilha@hotmail.com

[b] Residente em Fisioterapia Neurofuncional - Adulto no Hospital Universitário Regional do Norte do Paraná, Universidade Estadual de Londrina (UEL), Londrina, PR - Brasil, e-mail: biatcosta@hotmail.com

[c] Acadêmico do curso de graduação em Fisioterapia da Universidade Estadual de Londrina (UEL), Londrina, PR - Brasil, e-mail: bandeira_lima@hotmail.com

[d] Doutora em Fisiopatologia em Clínica Médica pela Faculdade de Medicina de Botucatu - UNESP, docente do curso de graduação em Fisioterapia da Universidade Estadual de Londrina (UEL), Londrina, PR - Brasil, e-mail: suhaila@uel.br

[e] Mestre em Ciências da Saúde pela Universidade Estadual de Londrina (UEL), docente do curso de graduação em Fisioterapia da Universidade Estadual de Londrina (UEL), Londrina, PR - Brasil, e-mail: spine@uel.br

\section{Resumo}

Introdução: Entre as consequências da lesão medular (LM), está o deficit na função sexual, o que interfere na qualidade de vida do indivíduo. Objetivos: Conhecer e descrever o perfil da sexualidade de homens com LM, comparando os períodos pré e pós-lesão. Materiais e métodos: Estudo transversal com 36 homens com LM. Os dados foram coletados através de um questionário (QSH-LM) e um roteiro. As variáveis quantitativas foram apresentadas por média e desvio padrão. As variáveis categóricas foram apresentadas por frequências absolutas e relativas e as associações foram realizadas através do teste Qui-quadrado. Todos os testes apresentaram significância de $5 \%$. Resultados: Houve prevalência de pacientes paraplégicos e de lesões do tipo completa. A prática, frequência, desejo e satisfação sexual decaíram após a LM, assim como as respostas sexuais. Dentre essas estão ereção, ejaculação e orgasmo, que decaíram de forma relevante após a LM. A sensação mais relatada durante o ato sexual foi o aumento da resposta cardiorrespiratória, antes ou após a LM, e mais indivíduos passaram a sentir nenhuma sensação durante a atividade sexual após a LM. Houve significativa associação entre tipo de lesão e presença de orgasmo e entre prática e satisfação sexual 
após a LM. Conclusão: Foi observado que a resposta sexual após a LM altera na ordem crescente: ereção, orgasmo e ejaculação. Percebe-se que muitas vezes a reabilitação é focada nas capacidades motoras e a sexualidade é pouco abordada.

Palavras-chave: Traumatismos da medula espinal. Sexualidade. Fisioterapia.

\section{Abstract}

Introduction: Among the consequences of spinal cord injury (SCI), there is the deficit in sexual function, which interferes in the quality of life of the individual. Objectives: To know and to describe the profile of the sexuality of men with SCI, comparing before and after injury periods. Materials and methods: Cross-sectional study with 36 men with SCI. Data were collected through a questionnaire (QSH-LM) and a screenplay. Quantitative variables were presented as mean and standard deviation. Categorical variables were presented by absolute and relative frequencies and associations were performed using the chi-square. All tests presented 5\% significance. Results: There was prevalence of paraplegic patients and complete lesions. The sexual practice, often, desire and satisfaction declined after SCI, as well as sexual responses, among these are erection, ejaculation and orgasm which declined materially after SCI. The most reported feeling during sex was the increasing cardiorespiratory response, before or after SCI, and more individuals began not to feel any sensation during sexual activity after SCI. There was a significant association between type of injury and the presence of orgasm and between sexual satisfaction and sexual practice after SCI. Conclusion: It was observed that sexual response after SCI changes in ascending order: erection, orgasm and ejaculation. It is noticed that the rehabilitation is often focused on motor skills and sexuality is rarely discussed.

Keywords: Spinal cord injuries. Sexuality. Physical therapy.

\section{Introdução}

Lesão medular (LM) é definida como qualquer comprometimento na medula espinal que acarrete deficits na função motora, sensitiva, visceral, além das funções sexuais do indivíduo (1). De acordo com a American Spinal Injury Association (ASIA), a ausência total das funções sensitiva e/ou motora e/ou autonômica abaixo do nível da lesão, inclusive nos níveis sacrais, caracteriza a lesão completa, enquanto a preservação de alguma função sensitiva e/ou motora e/ ou autonômica abaixo do nível da lesão, inclusive nos segmentos sacrais, caracteriza a lesão incompleta (1, 2). A incidência estimada de indivíduos com LM no Brasil é de 130 mil, enquanto a incidência mundial está entre 2,23 e 7,55 casos a cada 10 mil habitantes, número que vem aumentando em virtude dos acidentes automobilísticos $(1,2)$. A média de idade é de 33 anos, com prevalência no gênero masculino $(1,3)$.

Como o lesado medular apresenta deficiência ou ausência do sinergismo entre os sistemas que constituem o sistema nervoso autônomo, simpático e parassimpático, há consequências físicas para esse indivíduo, o qual relata o deficit na função sexual como primeira ou segunda principal queixa $(3,4)$. Tal fato se dá, principalmente, pela disfunção erétil, caracterizada como a incapacidade do homem atingir uma ereção suficientemente rígida para penetração vaginal e mantê-la por tempo satisfatório (5). Devese acrescentar também que a disfunção sexual inclui, além de mudanças físicas, neurais e endócrinas, o impacto sobre a autoimagem e as emoções do indivíduo com relação à sua sexualidade (6).

A resposta sexual após a LM depende da extensão e da localização da lesão (7). Portanto, pacientes com lesão na medula lombossacral (centro parassimpático) apresentarão a ereção psicogênica, a qual tem origem por estímulos visuais, auditivos, sentimentos ou fantasias, e pode ser menos rígida e manter-se por menos tempo que a ereção reflexa $(6,8)$. Pacientes com lesão entre o $2^{\circ}$ segmento medular torácico (T2) e o $2^{\underline{0}}$ segmento medular sacral (S2) apresentarão os centros simpático e parassimpático preservados, tendo assim a ereção psicogênica e reflexa, a qual se origina por estímulo sensorial e mantém-se pela tumescência peniana por tempo insatisfatório $(6,8)$. 
No entanto, na disfunção sexual após a LM, a ereção é menos prejudicada que a ejaculação, compreendida pela propulsão do sêmen ao sair pela uretra $(8,9)$. 0 orgasmo também é prejudicado consideravelmente, devido à disfunção erétil e à alteração da sensibilidade na lesão medular, porém ainda pode ser atingido com a exploração e estimulação de outras áreas erógenas ou fantasias pela ligação com fatores emocionais, mesmo que as sensações sejam diferentes e menos intensas das vividas antes da lesão (3). Dentre essas sensações, estão as alterações nas respostas cardiovasculares, musculares e autonômicas (10), assim como as sensações apresentadas em homens com a função sexual normal, na qual há aumento da frequência cardíaca e respiratória em conjunto com as contrações involuntárias da área peniana durante o orgasmo (11).

Para os homens, a diminuição da satisfação sexual se dá principalmente pela perda da motricidade voluntária, dificuldade para atingir o orgasmo, diminuição da intensidade do orgasmo e dificuldade em conseguir e manter a ereção, ou seja, sentem falta da resposta sexual típica que conheciam antes da LM, por centralizarem a sexualidade, principalmente, no pênis (12).

Considerando que o fisioterapeuta deve ter conhecimento sobre cada ponto do perfil do paciente com LM para uma programação mais eficaz e completa de sua reabilitação, este estudo tem por objetivo conhecer e descrever o perfil da sexualidade em homens com LM, comparando os períodos pré e pós-lesão, beneficiando tais homens que se encontram desamparados quanto ao conhecimento da atividade sexual na presente situação, além de contribuir para a literatura, visto que a sexualidade é um tema pouco abordado.

\section{Materiais e métodos}

Trata-se de um estudo transversal com 36 pacientes do gênero masculino, com 18 anos de idade ou mais, com o diagnóstico médico de LM, classificados em tetraplegia ou paraplegia, conforme padronização da ASIA, atendidos em clínicas e ambulatórios de fisioterapia na cidade de Londrina (PR), que concordaram em participar do estudo e assinaram o termo de consentimento livre e esclarecido. Não foram incluídos no estudo indivíduos não alfabetizados, em fase de choque medular, com disfunção sexual prévia à LM, com outras patologias associadas à LM, como traumatismo crânio encefálico ou mielopatias a esclarecer e hipótese diagnóstica de LM. Este estudo foi aprovado pelo Comitê de Ética e Pesquisa da Universidade Estadual de Londrina (UEL) com o Parecer n. 260/10 — "Grupo de Pesquisa: Avaliação e intervenção em Fisioterapia”, cadastrado no Conselho Nacional de Pesquisa (CNPq).

Os dados foram coletados por meio de dois instrumentos, um questionário e um roteiro. 0 primeiro instrumento contempla o Questionário de Sexualidade Humana na Lesão Medular (QSH-LM) (12), composto por 67 questões abertas e fechadas. 0 instrumento foi desenvolvido em três partes, contemplando os dados do participante e o perfil sexual nos períodos antes e após LM.

A primeira parte é composta pelas informações pessoais do participante, como: idade, dados sobre a LM e estado civil antes e após a LM. A segunda parte contempla as informações sobre sexualidade antes da LM, com quatro blocos de perguntas sobre: prática, orientação, satisfação e resposta sexual. Consideramos a prática sexual como sexo oral, anal, intercurso pênis-vagina e masturbação; orientação como o aconselhamento recebido pelo paciente e parceira; satisfação como o contentamento que o paciente apresenta pela sua vida sexual; e resposta sexual como as reações do paciente perante $o$ ato sexual. A terceira parte contempla as informações sobre a sexualidade após a lesão, com perguntas sobre aconselhamento sexual, além dos quatro blocos já citados.

No presente estudo, foram analisadas apenas 23 questões fechadas do referido questionário, das quais 7 são específicas para características pessoais e clínicas do participante, 14 para função sexual e 2 para orientação sexual. 0 segundo instrumento abordou 2 questões sobre as sensações apresentadas durante o ato sexual pré e pós-lesão medular.

Os participantes foram informados sobre a pesquisa e, no caso de aceitarem participar, assinaram o termo de consentimento livre e esclarecido. Receberam um envelope lacrado contendo o questionário e o roteiro. Foram orientados a preenchê-lo em seu domicílio e devolvê-lo, sem identificação, quando conveniente em uma urna disponibilizada no local de retirada.

As variáveis quantitativas foram apresentadas por média e desvio padrão após os pressupostos de 
normalidade serem atingidos pelo Teste de Shapiro Wilk. As variáveis categóricas foram apresentadas por meio de frequências absolutas e relativas e as associações foram realizadas através do teste Quiquadrado. As variáveis associadas foram: tipo de lesão e presença de orgasmo pós-LM, prática sexual pós-LM e satisfação sexual pós-LM, diagnóstico neurofuncional e prática sexual, presença de ereção, ejaculação e orgasmo pós-LM. A significância foi estipulada em $5 \%$ para todos os testes. Todos os dados foram tabulados e analisados no programa estatístico Statistical Package for the Social Sciences (SPSS ${ }^{\circledR}$ ), versão 20.

\section{Resultados}

Entre os 36 participantes, a média de idade encontrada foi de $36,64 \pm 9,95$ anos e a média de tempo de LM foi de 9,34 $\pm 7,31$ anos. A principal causa da LM foi acidente de trânsito, em 17 participantes $(47,2 \%)$, seguido de ferimento por arma de fogo em $13(36,1 \%)$, acidentes de trabalho em 3 (8,3\%), mergulho em águas rasas em $2(5,6 \%)$ e quedas em 1 $(2,8 \%)$. Quanto ao diagnóstico neurofuncional, 26 $(72,2 \%)$ apresentam o diagnóstico de paraplegia e $10(27,8 \%)$, tetraplegia. Em relação ao tipo de lesão, $20(55,6 \%)$ apresentaram LM do tipo completa e 16 $(44,4 \%)$ incompleta. Quanto ao estado civil antes da LM, 15 (41,7\%) eram casados, 11 (30,6\%) solteiros, 7 (19,4\%) estavam em união estável e 3 (8,3\%) eram separados; e após a LM 19 (52,8\%) são casados, 9 $(25,0 \%)$ solteiros, $5(13,9 \%)$ estão em união estável e $3(8,3 \%)$ estão separados.

Antes da LM, todos responderam ter vida sexual ativa, sendo que $11(30,6 \%)$ mantinham a frequência sexual de 3 vezes por semana, com média de 3,25 \pm 1,71 (Tabela 1). Todos tinham desejo sexual, sendo que $35(97,2 \%)$ eram satisfeitos sexualmente e 1 $(2,8 \%)$ não era. Dentre os participantes, 29 (80,5\%) apresentavam ereção espontânea antes do ato sexual e $6(16,7 \%)$ não apresentavam; todos conseguiam ejacular e chegar ao orgasmo antes da LM.

Após a LM, 27 (75,0\%) têm vida sexual ativa e 9 $(25,0 \%)$ não, sendo que $16(44,4 \%)$ mantem a frequência de menos de 1 vez por semana, com média de 1,56 $\pm 2,05$ vezes por semana (Tabela 1 ). Trinta e quatro $(94,4 \%)$ têm desejo sexual atualmente e 2 $(5,6 \%)$ não têm desejo, sendo que $29(80,6 \%)$ são satisfeitos sexualmente e $7(19,4 \%)$ se sentem nada satisfeitos. Dezoito $(50,0 \%)$ apresentam ereção espontânea antes do ato sexual, 14 (38,9\%) conseguem ejacular e $22(61,1 \%)$ não têm ejaculação. Dezesseis $(44,4 \%)$ conseguem chegar ao orgasmo e $20(55,6 \%)$ não.

Quanto às sensações apresentadas durante o ato sexual antes da LM, 17 (47,2\%) relataram aumento da frequência cardíaca, respiratória e da temperatura corporal; $11(30,6 \%)$ apresentavam aumento da frequência cardíaca, respiratória e da temperatura corporal e arrepio; 4 (11,1\%) sentiam apenas arrepio; e $4(11,1 \%)$ não apresentavam nenhuma sensação (Figura 1).

Quanto às sensações apresentadas durante o ato sexual após a LM, 12 (33,3\%) apresentam aumento da frequência cardíaca, respiratória e da temperatura corporal; $10(27,8 \%)$ não apresentam nenhuma sensação; $5(13,9 \%)$ apresentam aumento da frequência cardíaca, respiratória e da temperatura corporal, arrepio e espasticidade; $4(11,1 \%)$ relata aumento da frequência cardíaca, respiratória e da temperatura corporal e arrepio; 2 (5,6\%) apresentam aumento da frequência cardíaca, respiratória, da temperatura corporal e espasticidade; $1(2,8 \%)$ relata aumento da frequência cardíaca, respiratória, da temperatura corporal, dor e espasticidade; 1 (2,8\%) sente apenas arrepio; e $1(2,8 \%)$ sente arrepio e apresenta espasticidade (Figura 1).

Em relação ao aconselhamento sexual, 18 (50,0\%) não receberam qualquer tipo de orientação após a LM e 18 (50,0\%) receberam, sendo que 31 (86,1\%) concordam que tal aconselhamento melhoraria a qualidade da sua vida sexual e $5(13,9 \%)$ não.

Quando foi associado o tipo de LM com a presença de orgasmo (Tabela 2), houve diferença estatisticamente significativa $(\mathrm{p}=0,01)$ de que indivíduos com LM incompleta $(68,6 \%)$ conseguem atingir o orgasmo e pacientes com LM completa (75\%) não apresentam orgasmo.

Quanto à prática e satisfação sexual após a LM (Tabela 3), houve significativa associação de que todos os pacientes que tem prática sexual estão satisfeitos sexualmente $(\mathrm{p}<0,01)$.

Não houve diferença estatisticamente significativa de que o diagnóstico neurofuncional interfira na prática sexual $(\mathrm{p}=0,19)$, presença de ereção $(\mathrm{p}=0,45)$, ejaculação $(\mathrm{p}=0,14)$ ou orgasmo $(\mathrm{p}=$ $0,27)$ (Tabela 4). 
Tabela 1 - Frequência semanal da ativada sexual nos períodos pré e pós-LM

\begin{tabular}{lcc}
\hline & Antes da LM & Após LM \\
\hline Menos de 1 vez por semana & $1(2,8 \%)$ & $16(44,4 \%)$ \\
1 vez por semana & $5(13,9 \%)$ & $4(11,1 \%)$ \\
2 vezes por semana & $5(13,9 \%)$ & $10(27,8 \%)$ \\
3 vezes por semana & $11(30,6 \%)$ & $2(5,6 \%)$ \\
4 vezes por semana & $7(19,4 \%)$ & - \\
5 vezes por semana & $4(11,1 \%)$ & $1(2,8 \%)$ \\
7 vezes por semana & $3(8,3 \%)$ & $3(8,3 \%)$ \\
\hline
\end{tabular}

Fonte: Dados da pesquisa.

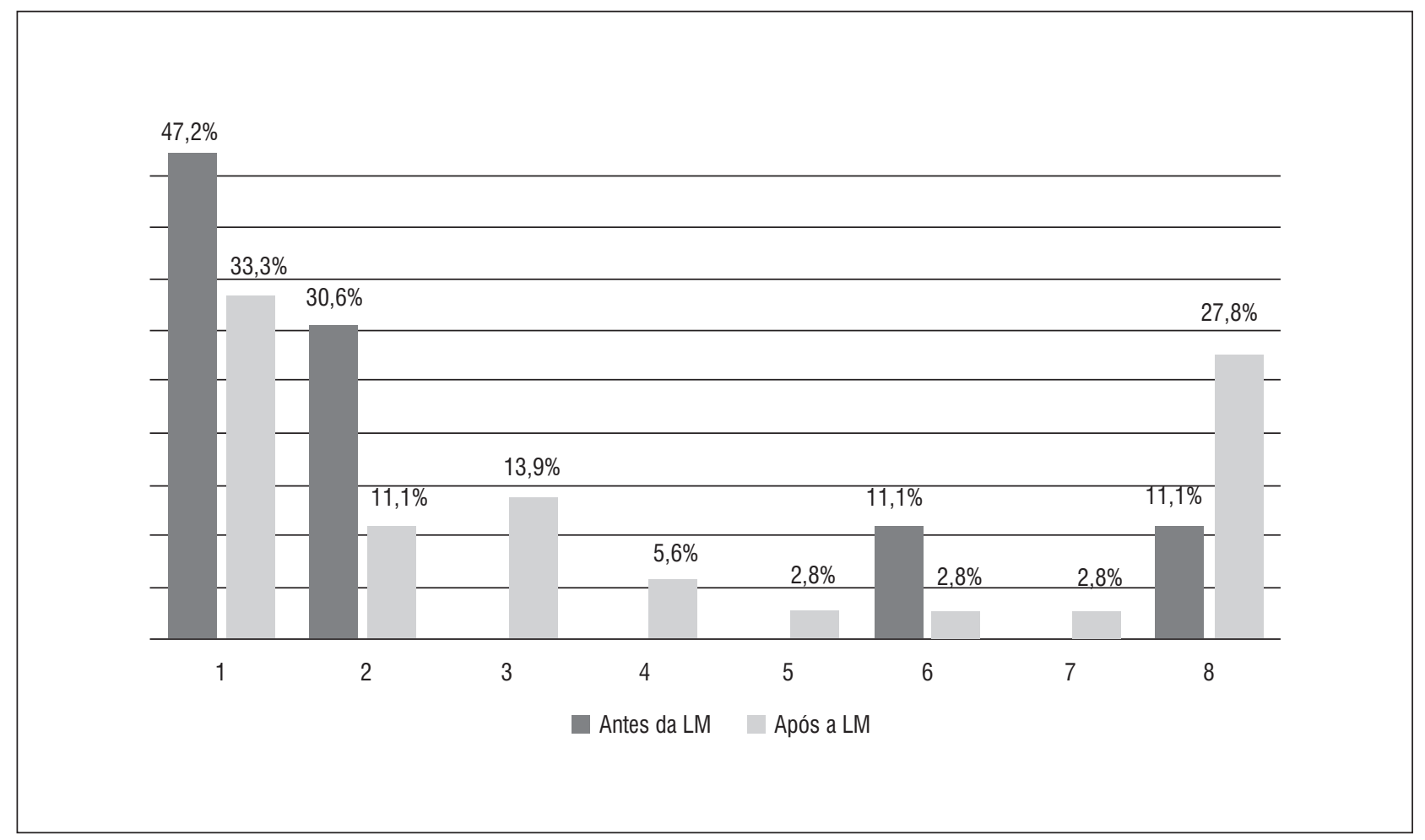

Figura 1 - Sensações apresentadas durante 0 ato sexual antes e após a LM

Legenda: 1 - Aumento da frequência cardíaca, respiratória e temperatura corporal; 2 - Aumento da frequência cardíaca, respiratória e temperatura corporal e arrepio; 3 - Aumento da frequência cardíaca, respiratória e temperatura corporal, arrepio e espasticidade; 4 - Aumento da frequência cardíaca, respiratória e temperatura corporal e espasticidade; 5 - Aumento da frequência cardíaca, respiratória e temperatura corporal, espasticidade e dor; 6 - Arrepio; 7 - Arrepio e espasticidade; 8 - Nenhuma.

Fonte: Dados da pesquisa. 
Tabela 2 - A presença de orgasmo quanto ao tipo de lesão

\begin{tabular}{lcc}
\hline & \multicolumn{2}{c}{ Orgasmo pós-LM } \\
\cline { 2 - 3 } Tipo de lesão & Não & Sim \\
\hline Incompleta $\mathrm{n}(\%)$ & $5(31,3 \%)$ & $11(68,8 \%)$ \\
Completa $\mathrm{n}(\%)$ & $15(75,0 \%)$ & $5(25,0 \%)$ \\
\hline
\end{tabular}

Fonte: Dados da pesquisa.

Tabela 3 - A satisfação sexual quanto à prática sexual após a LM

\begin{tabular}{lcc}
\hline & \multicolumn{2}{c}{ Satisfação sexual pós-LM } \\
\cline { 2 - 3 } Prática sexual pós-LM & Não & Sim \\
\hline Não $n(\%)$ & $7(77,8 \%)$ & $2(22,2 \%)$ \\
$\operatorname{Sim~} n(\%)$ & - & $27(100,0 \%)$ \\
\hline
\end{tabular}

Fonte: Dados da pesquisa.

Tabela 4 - A satisfação sexual quanto à prática sexual após a LM

\begin{tabular}{lccc}
\hline & \multicolumn{3}{c}{ Diagnóstico neurofuncional } \\
\cline { 2 - 4 } & Tetraplegia $\mathbf{n}=\mathbf{1 0}$ & Paraplegia $\mathbf{n}=\mathbf{2 6}$ \\
\hline Prática sexual n (\%) & $6(60,0 \%)$ & $21(80,8 \%)$ & $\mathbf{P}, 19$ \\
Ereção n (\%) & $4(40,0 \%)$ & $14(53,8 \%)$ & 0,45 \\
Ejaculação n (\%) & $2(20,0 \%)$ & $12(46,2 \%)$ & 0,14 \\
Orgasmo n (\%) & $3(30,0 \%)$ & $13(50,0 \%)$ & 0,27 \\
\hline
\end{tabular}

Fonte: Dados da pesquisa.

\section{Discussão}

A média de idade foi de 36,94 anos, idade que se enquadra na classificação etária da população ativa, semelhante à literatura $(1,3,13)$, porém em contradição com a revisão sistemática (14), que observou diferente distribuição etária em pacientes com LM, um pico entre 15 e 29 anos e outro pico acima dos 65 anos de idade. As principais causas da LM foram acidentes de trânsito $(47,2 \%)$ e violência $(36,1 \%)$, dados semelhantes encontrados em outros estudos $(2,14,15)$, porém discordantes de outros autores, que afirmam ser a violência a principal causa $(13,16)$. DeVivo et al. (15) afirmam que a grande incidência de lesados medulares devido à acidentes de trânsito é explicada pelo fato de os cidadãos permanecerem tempos prolongados em meios de transporte, realizarem pouco uso do cinto de segurança, terem hábitos inadequados de dirigir e trafegar em estradas em más condições.

Como a disfunção sexual está diretamente relacionada ao nível da lesão e ao tempo pós-lesão (17), tais dados foram avaliados no presente estudo. Foi encontrada a prevalência de paraplegia $(72,2 \%)$ e LM completa $(55,6 \%)$, prevalência também citada na literatura $(3,13)$. A média do tempo de LM nos indivíduos deste estudo é de 9,28 anos, sendo este dado considerado um fator determinante para a prática 
sexual, pois o paciente necessita de determinado período para o reajuste, não só físico, como a fase de choque medular, mas psicoemocional, visto que ele passa por um período de assexualidade no início, seguido por um período de redescoberta durante a reabilitação (3).

Quanto ao estado civil, o número de participantes casados antes da LM aumentou em relação ao período após a LM, de $41,7 \%$ para 52,8\%, e o contrário ocorreu com os solteiros, que de $30,6 \%$ diminuiu para $25,0 \%$, semelhante ao estudo de Magalhães et al. (1), o qual evidenciou que $57,1 \%$ são casados enquanto $35,7 \%$ são solteiros. Quando o casamento ocorre no período após LM, há maior probabilidade de sucesso da união, pois o relacionamento inicia com as limitações estabelecidas e até mesmo o parceiro que apresenta LM se encontra já adaptado à sua condição, diferente do casamento que ocorre antes da LM, no qual o parceiro sofre o desgaste emocional da fase aguda da lesão, vivenciando os reajustes necessários (18).

Antes da LM, todos tinham vida sexual ativa, e depois, 75\% mantêm essa prática, como no estudo de Dahlberg et al. (19), com $76 \%$ e acima do relatado por Reitz et al. (20), segundo os quais $57,5 \%$ dos pacientes têm vida sexual ativa, com sua frequência reduzida após a $\operatorname{LM}(3,20)$, assim como relatado no presente estudo, no qual a média da prática sexual semanal decaiu de 3,25 para 1,56 vezes por semana. A prática e a frequência sexual ficam reduzidas após a LM, sendo um dos motivos considerados a orientação superficial que o paciente e sua parceira recebem sobre sexualidade, dificultando o reajuste sexual do casal (12). Ainda se deve acrescentar que a resposta erétil é muitas vezes insuficiente para experimentar o coito e não surge sempre que desejada ou com duração suficiente. Esses fatores, junto com o medo de rejeição ou fracasso, contribuem para a redução da frequência da atividade sexual (21).

Dentre os fatores que influenciam a diminuição da satisfação sexual no período pós-LM, está o prejuízo sobre a sensibilidade, a mobilidade, o orgasmo e a baixa autoestima (22). Mendes et al. (23) relataram que a satisfação sexual reduziu de $80 \%$ antes da LM para 47,5\% após a LM, enquanto Reitz et al. (20) relataram que apenas 38\% estão satisfeitos com sua vida sexual. Na pesquisa atual, $97,2 \%$ estavam satisfeitos sexualmente e todos tinham desejo sexual antes da LM, e depois, 80,6\% estão satisfeitos sexualmente e $94,4 \%$ mantem o desejo sexual. Esse resultado contrastante com a literatura se deve a fatores ambientais e socioculturais, fatores esses que influenciam no desempenho sexual, considerado por homens como mais importante que a aparência física $(24,25)$. 0 estudo de Biering-Sørensen et al. (26) acrescenta que os pacientes relacionam a diminuição da satisfação sexual com problemas intestinais e urinários, úlceras de pressão, espasticidade e dor.

Estudos $(26,27)$ evidenciaram que $70 \%$ a $80 \%$ de indivíduos com LM há mais de seis meses atingem a ereção, sendo esses indivíduos mais jovens (26), enquanto o estudo de Baasch (12) citou que apenas $28,6 \%$ dos indivíduos têm ereção sempre ou muitas vezes. Já no presente estudo, observou-se que 50\% dos participantes apresentam ereção espontânea após a LM; antes, eram $83,3 \%$. Essa perda relevante ocorre em virtude da lesão e da interrupção das vias da medula espinal, incapacitando o cérebro de efetuar a modulação do processo da ereção. E mesmo quando o indivíduo consegue atingir a ereção, esta pode não ter rigidez e duração suficientes para a penetração (27), considerando que os aspectos psicológicos também são fatores condicionantes da ereção (21).

No presente estudo, todos conseguiam ejacular e chegar ao orgasmo antes da LM, porém essas capacidades foram prejudicadas após a LM, sendo que $38,9 \%$ conseguem ejacular e $44,4 \%$ conseguem chegar ao orgasmo, dados semelhantes aos da literatura $(3,9,20,26)$. Esse decréscimo se explica pelo fato da LM prejudicar o sinergismo do sistema nervoso, dificultando a ejaculação, pois há perda da contração rítmica do músculo bulbocavernoso do pênis (27). É notável que a capacidade de obter um orgasmo também diminui consideravelmente após a LM, pela perda dos elementos sensoriais dos órgãos genitais e efeitos fisiológicos (19), considerando que os pacientes reportam algo semelhante ao orgasmo, como sensações diferentes e menos intensas quando comparadas às sensações vividas no período pré-lesão (3).

Durante a relação sexual, há uma reação generalizada do organismo, que responde com o aumento da pressão arterial e da frequência cardíaca e respiratória (5), sendo assim, esse foi o dado mais encontrado entre as sensações apresentadas durante 0 ato sexual, seja antes ou após a LM. Dentre as sensações relatadas, o aumento da frequência cardíaca, respiratória e da temperatura corporal foi citado por $44,4 \%$ dos indivíduos e 11,1\% não apresentavam alguma sensação antes da lesão, enquanto 33,3\% apresentam aumento 
da frequência cardíaca, respiratória e da temperatura corporal, 27,8\% não apresentam qualquer sensação e 13,9\% apresentam aumento da frequência cardíaca, respiratória e da temperatura corporal, arrepio e espasticidade durante o ato sexual após a LM.

Essa diferença de adaptação cardiovascular durante a atividade sexual, entre os períodos pré e pós-lesão, dá-se pelo reduzido estímulo simpático no lesado medular, o que limita tal adaptação (21). Observa-se também que os indivíduos relataram sensações como arrepio e espasticidade, o que foi encontrado com frequência no estudo de Magalhães (21), no qual os participantes descreveram o orgasmo como um súbito aumento da espasticidade, seguido de um relaxamento muscular global, sensações de prazer na região do abdômen, pelve e coxas e, ocasionalmente, sensações dolorosas. Dados semelhantes foram relatados por Anderson et al. (4), onde 35\% apresentam espasticidade durante o ato sexual e 38,8\% não referem sensação alguma.

Observou-se que 50\% receberam algum tipo de orientação ou aconselhamento sexual após a LM e $86,1 \%$ concordam que a orientação melhoraria sua vida sexual. Em outro estudo, (12) 70,2\% receberam orientação e $50 \%$ acreditam que o aconselhamento ajudaria na vida sexual. 0 dado mostrado evidencia o interesse da população do presente estudo em buscar alternativas para a melhor qualidade da vida sexual.

Evidenciou-se um valor estatisticamente significativo que $68,8 \%$ dos pacientes com lesão do tipo incompleta conseguem atingir o orgasmo $(p=0,01)$, pois eles apresentam menor comprometimento sensitivo e motor, quando comparados com os pacientes com lesão completa, o que ajuda na repercussão dos fatores psicológicos. Também se constatou significativamente que todos os pacientes que têm prática sexual são satisfeitos sexualmente $(\mathrm{p}<0,01)$, pois é visto que a satisfação é mais relacionada aos papéis sociais do que ao prejuízo físico (18), ou seja, o homem se satisfaz ao contribuir com sua virilidade, independente de sua inabilidade.

Embora não estatisticamente significativo, dentre os pacientes paraplégicos, $80,8 \%$ têm vida sexual ativa $(p=0,19), 53,8 \%$ têm ereção espontânea antes do ato sexual $(p=0,45), 46,2 \%$ conseguem ejacular $(p=$ $0,14)$ e $50,0 \%$ atingem o orgasmo $(p=0,27)$. Tal fato explica-se por esses pacientes serem mais ativos, na maioria dos casos, por sua maior capacidade de mobilidade e menor comprometimento de sensibilidade, 0 que contribui para melhor desempenho na atividade sexual. Everaert et al. (9) também constataram que lesões mais baixas têm maior percentual de preservação da ejaculação, presente em $18 \%$ dos paraplégicos de seu estudo, enquanto $4 \%$ dos indivíduos com lesões mais altas conseguem ter ejaculação, e o orgasmo não está relacionado com o nível da lesão.

Acredita-se que um fator importante desta pesquisa foi obter conhecimento sobre as sensações que o paciente apresentava e apresenta durante o ato sexual antes e após a LM, uma vez que muitos pacientes e profissionais da saúde desconheciam tais sinais e sintomas, em virtude da escassez desses dados na literatura. Tais informações são relevantes para a equipe multiprofissional incrementar e direcionar o programa de reabilitação, para o paciente ter reconhecimento de seu corpo e para seu cônjuge se adaptar mais facilmente às várias mudanças agora estabelecidas e aceitá-las. Com isso, o estudo contribui para o despertar da consciência da população quanto à atividade sexual na LM, acreditando que esta é possível, dependendo das limitações de cada indivíduo. Considera-se que o presente estudo foi limitado pela não aceitação de alguns lesados medulares contatados em participar da pesquisa, diminuindo o número de participantes e interferindo no poder de generalização dos resultados.

\section{Conclusão}

A resposta sexual altera-se após a LM graças à perturbação ou interrupção das vias de comunicação necessárias para isso. Assim, as respostas foram prejudicadas na ordem crescente: ereção, orgasmo e ejaculação. E com isso, há consequente redução da prática, frequência, desejo e satisfação sexual.

Constatou-se que os relatos de sensações durante o ato sexual decaíram após a lesão, em virtude da alteração de sensibilidade que o indivíduo apresenta. Porém, deve-se lembrar de que na fase de reabilitação o paciente pode substituir essa sensibilidade perineal ausente ou deficiente por novas áreas erógenas. Evidenciou-se, estatisticamente significante, que lesados medulares com lesão incompleta tem a capacidade de obter orgasmo e que indivíduos que têm prática sexual são satisfeitos sexualmente.

0 conhecimento sobre $o$ assunto sexualidade na lesão medular não está bem difundido entre os pacientes e os terapeutas. Graças a isso os participantes se mostraram interessados em receber um aconselhamento 
sexual, buscando melhor qualidade de vida. É importante realçar, para esses pacientes, que a deficiência física não neutraliza a sexualidade, mostrando o que pode ser feito para o melhor desempenho na atividade sexual, de acordo com a limitação de cada indivíduo.

Muitas vezes, a reabilitação é focada nas capacidades motoras para promoção de um bem-estar físico, porém a sexualidade é pouco abordada para promoção também do bem-estar emocional. Para isso, devem-se elaborar orientações multiprofissionais e aconselhamentos sobre a atividade sexual para os homens com LM e suas parceiras, com o objetivo de ampliar o conhecimento sobre tal assunto no ponto de vista do profissional e do paciente, contribuindo para uma reabilitação mais eficaz.

\section{Referências}

1. Magalhães MO, Sousa ANB, Costa LOP, Pinto DS. Avaliação em pacientes com traumatismo raquimedular: um estudo descritivo e transversal. ConScientiae Saúde. $2011 ; 10(1): 69-76$.

2. França ISX, Coura AS, França EG, Basilio NNV, Souto RQ. Qualidade de vida de adultos com lesão medular: um estudo com WHOQOL-bref. Rev Esc Enferm USP. 2011;45(6):1364-71.

3. Garrett A, Martins F, Teixeira Z. Programa de intervenção para a reabilitação da sexualidade numa população portuguesa de lesionados medulares. In: Actas do 7. Simpósio Nacional de Investigação em Psicologia, 4-6 fev 2010; Braga. Braga: Universidade do Minho; 2010.

4. Anderson KD, Borisoff JF, Johnson RD, Stiens AS, Elliott SL. The impact of spinal cord injury on sexual function: concerns of the general population. Spinal Cord. 2007;45(5):328-37.

5. Barbosa VRC. A vivência da sexualidade de homens com lesão medular adquirida [dissertação]. São Paulo: Universidade de São Paulo; 2003.

6. Ramos AS, Samsó JV. Specific aspects of erectile dysfunction in spinal cord injury. Int J Impot Res. 2004;16(Suppl 2):S42-5.

7. DeForge D, Blackmar J, Garrity C, Yazdi F, Cronin V, Barrowman N, et al. Male erectile dysfunction following spinal cord injury: a systematic review. Spinal Cord. 2006;44(8):465-73.
8. Alexander MS, Biering-Sorensen F, Elliott S, Kreuter M, Sonksen J. International spinal cord injury male sexual function basic data set. Spinal Cord. 2011;49(7):795-8.

9. Everaert K, Waard WIC, Hoof TV, Kiekens C, Mulliez T, D'Herde C. Neuroanatomy and neurophysiology related to sexual dysfunction in male neurogenic patients with lesions to the spinal cord or peripheral nerves. Spinal Cord. 2010;48(3):182-191.

10. Courtois F, Charvier K, Vézina JG, Journel NM, Carrier S, Jacquemin G, et al. Assessing and conceptualizing orgasm after a spinal cord injury. BJU International. 2011;108(10):1624-33.

11. Chia M, Chia M, Abrams D, Abrams RC. O orgasmo múltiplo do casal: prazer, intimidade e saúde - como aproveitar ao máximo a vida a dois. Trad. Neuza Capelo. Rio de Janeiro: Objetiva; 2003.

12. Baasch AKM. Sexualidade na lesão medular [dissertação]. Florianópolis: Universidade do Estado de Santa Catarina; 2008.

13. Silva RA. Condições de funcionalidade de pessoas com lesão medular fundamentadas no índice de Barthel: proposta de intervenção de enfermagem [dissertação]. Fortaleza: Universidade Federal do Ceará; 2011.

14. van den Berg MEL, Castellote JM, Mahillo-Fernandez I, Pedro-Cuesta J. Incidence of spinal cord injury worldwide: a systematic review. Neuroepidemiology. 2010;34(3):184-92.

15. DeVivo MJ. Epidemiology of traumatic spinal cord injury: trends and future implications. Spinal Cord. 2012;50(5):365-72.

16. Schoeller SD, Borges AMF, Brignol P, Kuhnen AE. Conhecer para cuidar: características de pessoas com lesão medular atendidas em um centro de reabilitação. R pesq cuid fundam. 2012;4(3):2598-604.

17. Scussel MM. A fisioterapia na reabilitação sexual em pacientes com lesão medular do município de Criciúma-SC [dissertação]. Criciúma: Universidade do Extremo Sul Catarinense; 2011.

18. Murta SG, Guimarães SS. Enfrentamento à lesão medular traumática. Estud psicol. 2007;12(1):57-63.

19. Dahlberg A, Alaranta H, Kautiainen H, Kotila M. Sexual activity and satisfaction in men with traumatic spinal cord lesion. Rehabil Med. 2007;39:152-55. 
20. Reitz A, Tobe V, Knapp PA, Schurch B. Impact of spinal cord injury on sexual health and quality of life. Int J Impot Res. 2004;16(2):167-74.

21. Magalhães IMB. Influência da actividade física na sexualidade de indivíduos com lesão vértebro-medular [dissertação]. Porto: Universidade do Porto; 2005.

22. Abromson CE, McBride KE, Konnyu KJ, Elliott SL; SCIRE Research Team. Sexual health outcome measures for individuals with a spinal cord injury: a systematic review. Spinal Cord. 2008;46(5):320-4.

23. Mendes AK, Cardoso FL, Savall ACR. Sexual satisfaction in people with spinal cord injury. Sex Disabil. 2008;26(3):137-47.

24. Araújo AA, Brito AM, Ferreira MNL, Petribú K, Mariano MHA. Modificações da qualidade de vida sexual de obesos submetidos à cirurgia de Fobi-Capella. Rev Col Bras Cir. 2009;36(1):42-8.

25. Cardoso FL, Savall AC, Sabbag S, Mendes AK, Beltrame TS. Implicações do conhecimento corporal no comportamento sexual. Rev bras educ fís esporte. 2009; 23(4):345-54.
26. Biering-Sørensen I, Hansen RB, Biering-Sørensen F. Sexual function in a traumatic spinal cord injured population 10-45 years after injury. J Rehabil Med. 2012;44(11):926-31.

27. Dimitriadis F, Karakitsios K, Tsounapi P, Tsambalas S, Loutradis D, Kanakas N, et al. Erectile function and male reproduction in men with spinal cord injury: a review. Andrologia. 2010;42(3):139-65.

Recebido: 07/01/2013

Received: $01 / 07 / 2013$

Aprovado: 22/08/2013

Approved: 08/22/2013 